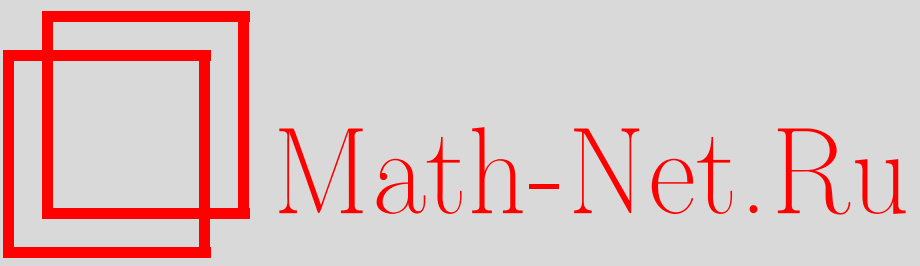

П. Р. Гордоа, Алгебраические и дифференциальные нелинейные формулы суперпозиции, ТМФ, 2003, том 137, номер 1, 87-96

DOI: https://doi.org/10.4213/tmf247

Использование Общероссийского математического портала Math-Net.Ru подразумевает, что вы прочитали и согласны с пользовательским соглашением

http://www.mathnet.ru/rus/agreement

Параметры загрузки:

IP: 54.197 .217 .227

26 апреля 2023 г., $14: 41: 37$ 
ТЕОРЕТИЧЕСКАЯ

И МАТЕМАТИЧЕСКАЯ

ФИЗИКА

Том 137, № 1

октябрь, 2003

(C) 2003 г.

П.Р. Гордоа*

\section{АЛГЕБРАИЧЕСКИЕ И ДИФФЕРЕНЦИАЛЬНЫЕ НЕЛИНЕЙНЫЕ ФОРМУЛЫ СУПЕРПОЗИЦИИ}

Знание пары Лакса и преобразования Дарбу вполне интегрируемой системы позволяет строить точные решения при помощи итерационной процедуры. Такой подход предполагает нахождение собственной функции пары Лакса на каждом шаге. Однако этот процесс можно значительно упростить, пользуясь преобразованием Беклунда и теоремой Бианки о перестановочности. С их помощью удается получить так называемую нелинейную формулу суперпозиции, позволяющую строить новое решение системы из трех имеющихся. Преимущество этого подхода состоит в том, что нелинейные формулы суперпозиции содержат производные низшего порядка по сравнению с парами Лакса и в некоторых случаях сводятся к алгебраическим уравнениям. Мы рассматриваем конструкцию новых нелинейных формул суперпозиции как в виде дифференциальных, так и в виде алгебраических уравнений.

Ключевые слова: нелинейная формула суперпозиции, преобразования Беклунда.

\section{1. ВВЕДЕНИЕ}

Итерация преобразования Дарбу (ПД) является, конечно, известным методом построения точных решений заданного полностью интегрируемого дифференциального уравнения в частных производных (УЧП). Такой подход предполагает нахож дение собственной функции пары Лакса (т.е. решение дифференциального уравнения) на каждом шаге итерационного процесса. Итерационный метод можно упростить, воспользовавшись преобразованием Беклунда (ПБ), которое нетрудно получить, исключая собственную функцию пары Лакса при помощи ПД. При этом теорема о перестановочности позволяет получить так называемую нелинейную формулу суперпозиции - выражение, позволяющее строить новое решение рассматриваемой системы из трех имеющихся. Преимушество этого подхода состоит в том, что нелинейные формулы суперпозиции содержат производные низшего порядка по сравнению с парами Лакса и в некоторых случаях даже сводятся к алгебраическим уравнениям. Алгебраические нелинейные формулы суперпозиции известны, например, для уравнения Кортевега-де Фриза и уравнения синус-Гордон (см., например, [1]). Некоторые примеры дифференциальных

*Area de Física Teórica, Facultad de Ciencias, Universidad de Salamanca, 37008 Salamanca, Spain. E-mail: prg@sonia.fis.usal.es 
нелинейных формул суперпозиции для уравнений с парами Лакса высшего порядка были недавно найдены в работе [2]. Отметим еще, что нелинейные формулы суперпозиции можно строить также при помощи билинейного формализма (см., например, [3]).

В данной статье рассматривается построение нелинейных формул суперпозиции для двух интегрируемых систем. В качестве простейшего примера укажем, что эти нелинейные формулы суперпозиции могут быть использованы при построении двухсолитонного решения. Отметим вместе с тем, что их можно использовать и для других целей, например, для построения новых решений, не описывающихся известной $N$-солитонной формулой, путем комбинации решений разного типа.

Статья имеет следуюшую структуру. В разделе 2 выводится алгебраическая нелинейная формула суперпозиции для системы двух связанных уравнений. В разделе 3 строится дифференциальная нелинейная формула суперпозиции для скалярного уравнения; эта формула представляет собой линейное дифференциальное уравнение третьего порядка. В том же разделе мы рассмотрим редукции этого скалярного уравнения к обыкновенным дифференциальным уравнениям (ОДУ) при помощи групп Ли его симметрий, в том числе редукцию к частным случаям пятого уравнения Пенлеве. В разделе 4 приведено короткое резюме полученных результатов.

\section{2. АЛГЕБРАИЧЕСКАЯ НЕЛИНЕЙНАЯ ФОРМУЛА СУПЕРПОЗИЦИИ}

В данном разделе мы приводим пример интегрируемой системы, для которой нелинейная формула суперпозиции сводится к алгебраическому выражению, позволяюшему строить новое решение системы из трех имеющихся. Такая система может быть записана в виде

$$
\begin{gathered}
u_{x x}+v_{t x}+\frac{1}{2}\left(u_{x} u_{t}\right)_{x}=0 \\
v_{x x}+u_{t x x x}+v_{x} u_{t x}+\frac{1}{2}\left(u_{x} v_{t x}+u_{t} v_{x x}\right)=0
\end{gathered}
$$

и соответствует первому отрицательному потоку классической иерархии Бусине. Эта система была введена в работе [4], там же приведены ее пара Лакса, ПД и односолитонное решение. Система (1) возникает как условие согласования для пары Лакса второго порядка

$$
\begin{aligned}
\psi_{x x} & =\frac{1}{2}\left(u_{x}-2 \lambda\right) \psi_{x}-\frac{1}{4}\left(v-u_{x}\right)_{x} \psi, \\
\psi_{t} & =-\frac{1}{2 \lambda}\left(u_{t}+2\right) \psi_{x}-\frac{1}{4 \lambda}\left(v-u_{x}\right)_{t} \psi,
\end{aligned}
$$

где $\lambda$ - постоянный спектральный параметр. Приведенное в работе [4] ПД системы (1) имеет вид

$$
\begin{gathered}
\tilde{u}=u+2 \ln \frac{\psi}{\psi_{x}} \\
\tilde{v}=v-u_{x}+2\left[\frac{\psi_{x}}{\psi}-\frac{1}{4}\left(v-u_{x}\right)_{x} \frac{\psi}{\psi_{x}}\right] .
\end{gathered}
$$


Оно связывает два решения $(u, v)$ и $(\tilde{u}, \tilde{v})$ системы $(1)$. Для вывода нелинейной формулы суперпозиции прежде всего построим ПБ для системы (1). Для этого исключим $\psi$ из пары Лакса (2) и первого уравнения в ПД (3). В результате получаем

$$
\begin{gathered}
p_{x}+p^{2}-\frac{1}{2}\left(u_{x}-2 \lambda\right) p+\frac{1}{4}\left(v-u_{x}\right)_{x}=0, \\
p_{t}+\frac{1}{2 \lambda} u_{t x} p+\frac{1}{2 \lambda}\left(u_{t}+2\right) p_{x}+\frac{1}{4 \lambda}\left(v-u_{x}\right)_{t x}=0,
\end{gathered}
$$

где $p=e^{-(\tilde{u}-u) / 2}=\psi_{x} / \psi$. При помощи второго уравнения в ПД (3) нетрудно выразить новое решение $\tilde{v}$ в терминах $p$ (и, следовательно, $\tilde{u})$ :

$$
\tilde{v}=v-u_{x}+2 p-\frac{1}{2}\left(v-u_{x}\right)_{x} \frac{1}{p} .
$$

Задача свелась к нахождению выражения для $\tilde{u}$.

Рассмотрим теперь уравнение (4) - пространственную часть ПБ. Предположим, что два решения $\left(u_{j, 1}, v_{j, 1}\right)$ и $\left(u_{j, 2}, v_{j, 2}\right)$ системы (1) построены на основе одного и того же исходного решения $\left(u_{j-1}, v_{j-1}\right)$ при помоши преобразований с (различными) спектральными параметрами $\lambda_{1}$ и $\lambda_{2}$, соответственно. Тогда имеем две различные копии уравнения (4) - одну с $p=e^{-\left(u_{j, 1}-u_{j-1}\right) / 2}$ и $\lambda=\lambda_{1}$, а другую с $p=e^{-\left(u_{j, 2}-u_{j-1}\right) / 2}$ и $\lambda=\lambda_{2}-$ имеющие вид

$$
\begin{aligned}
& \left(u_{j, 1}\right)_{x}=2 e^{-\left(u_{j, 1}-u_{j-1}\right) / 2}+\frac{1}{2}\left[v_{j-1}-\left(u_{j-1}\right)_{x}\right]_{x} e^{\left(u_{j, 1}-u_{j-1}\right) / 2}+2 \lambda_{1}, \\
& \left(u_{j, 2}\right)_{x}=2 e^{-\left(u_{j, 2}-u_{j-1}\right) / 2}+\frac{1}{2}\left[v_{j-1}-\left(u_{j-1}\right)_{x}\right]_{x} e^{\left(u_{j, 2}-u_{j-1}\right) / 2}+2 \lambda_{2} .
\end{aligned}
$$

Предположим теперь, что исходя из $\left(u_{j, 1}, v_{j, 1}\right)$ построено новое решение $\left(u_{j+1,12}\right.$, $\left.v_{j+1,12}\right)$ со спектральным параметром $\lambda_{2}$, а из $\left(u_{j, 2}, v_{j, 2}\right)$ - решение $\left(u_{j+1,21}, v_{j+1,21}\right)$ со спектральным параметром $\lambda_{1}$. Как и раньше, имеем две копии уравнения (4) (одну с $p=e^{-\left(u_{j+1,12}-u_{j, 1}\right) / 2}$ и $\lambda=\lambda_{2}$, а другую с $p=e^{-\left(u_{j+1,21}-u_{j, 2}\right) / 2}$ и $\left.\lambda=\lambda_{1}\right):$

$$
\begin{aligned}
& \left(u_{j+1,12}\right)_{x}=2 e^{-\left(u_{j+1,12}-u_{j, 1}\right) / 2}+\frac{1}{2}\left[v_{j, 1}-\left(u_{j, 1}\right)_{x}\right]_{x} e^{\left(u_{j+1,12}-u_{j, 1}\right) / 2}+2 \lambda_{2}, \\
& \left(u_{j+1,21}\right)_{x}=2 e^{-\left(u_{j+1,21}-u_{j, 2}\right) / 2}+\frac{1}{2}\left[v_{j, 2}-\left(u_{j, 2}\right)_{x}\right]_{x} e^{\left(u_{j+1,21}-u_{j, 2}\right) / 2}+2 \lambda_{1} .
\end{aligned}
$$

Воспользуемся теперь теоремой о перестановочности, утверждающей, что $u_{j+1,21}=$ $u_{j+1,12}$ (в дальнейшем для простоты полагаем $u_{j+1,21}=u_{j+1,12}=u_{j+1}$ ). Тогда можно исключить первую производную $u_{j+1}$ из уравнений $(9)$ и (10). В результате получаем

$$
\begin{aligned}
& G_{j+1}^{2}\left\{\left[v_{j, 1}-\left(u_{j, 1}\right)_{x}\right]_{x} G_{j, 1}^{-1}-\left[v_{j, 2}-\left(u_{j, 2}\right)_{x}\right]_{x} G_{j, 2}^{-1}\right\}+ \\
& +4 G_{j+1}\left(\lambda_{2}-\lambda_{1}\right)+4\left(G_{j, 1}-G_{j, 2}\right)=0
\end{aligned}
$$

где $G_{j, 1}=e^{u_{j, 1} / 2}, G_{j, 2}=e^{u_{j, 2} / 2}$ и $G_{j+1}=e^{u_{j+1} / 2}$. Таким образом можно получить новое решение $u_{j+1}$, просто решив квадратичное по $G_{j+1}$ уравнение $(11)$. 
Но этот полином можно еше упростить, воспользовавшись уравнением (6). Точно так же, как и в случае уравнения (4), имеем четыре различные копии этого уравнения. Первые две из них (с $p=e^{-\left(u_{j, 1}-u_{j-1}\right) / 2}$ и $\left.p=e^{-\left(u_{j, 2}-u_{j-1}\right) / 2}\right)$ вместе с уравнениями $(7)$ и (8) позволяют исключить производные $\left(u_{j, 1}, v_{j, 1}\right)$ и $\left(u_{j, 2}, v_{j, 2}\right)$ из $(11)$. В итоге находим, что уравнение (11) факторизуется:

$$
\begin{aligned}
& {\left[G_{j+1}-4 \frac{G_{j-1}}{\left(u_{j-1}\right)_{x x}-\left(v_{j-1}\right)_{x}}\right] \times} \\
& \quad \times\left[G_{j+1}+\frac{4 G_{j-1}\left(G_{j, 2}-G_{j, 1}\right)}{4 G_{j-1}\left(\lambda_{1}-\lambda_{2}\right)+\left(G_{j, 2}-G_{j, 1}\right)\left[\left(u_{j-1}\right)_{x x}-\left(v_{j-1}\right)_{x}\right]}\right]=0
\end{aligned}
$$

где $G_{j-1}=e^{u_{j-1} / 2}$. Первый сомножитель в (12) дает простое решение в терминах $\left(u_{j-1}, v_{j-1}\right)$ - только одного из трех предыдуших решений. В действительности именно второй сомножитель

$$
G_{j+1}=G_{j-1}\left[\frac{\left(\lambda_{2}-\lambda_{1}\right)}{\left(G_{j, 2}-G_{j, 1}\right)} G_{j-1}-\frac{\left[\left(u_{j-1}\right)_{x x}-\left(v_{j-1}\right)_{x}\right]}{4}\right]^{-1}
$$

позволяет строить решения при помощи итераций. Подставляя $G_{j+1}=e^{u_{j+1} / 2}$ и $G_{j-1}=$ $e^{u_{j-1} / 2}$, получаем алгебраическое соотношение

$$
u_{j+1}=u_{j-1}-2 \ln \left[\frac{\left(\lambda_{2}-\lambda_{1}\right)}{\left(G_{j, 2}-G_{j, 1}\right)} G_{j-1}-\frac{\left[\left(u_{j-1}\right)_{x x}-\left(v_{j-1}\right)_{x}\right]}{4}\right],
$$

выражающее $u_{j+1}$ в терминах известных решений $\left(u_{j-1}, v_{j-1}\right),\left(u_{j, 1}, v_{j, 1}\right)$ и $\left(u_{j, 2}, v_{j, 2}\right)$ системы (1). Выражение (14) представляет собой нелинейную формулу суперпозиции для системы (1) и позволяет строить решения этой системы при помощи итераций, не решая дифференциальное уравнение. Отметим также, что в этом процессе используется только пространственная часть ПБ. Это означает, что нелинейная формула суперпозиции (14) справедлива для всей классической иерархии Буссинеска (подробнее об этой иерархии см. [4], [5]). Нелинейная формула суперпозиции (14) использовалась в работе [6] для получения обшего вида двухсолитонного решения системы (1), которое содержит произвольную функцию времени. Наличие этой функции позволяет получить богатый запас солитонных взаимодействий, в том числе такие новые явления, как процессы повторного расщепления и слияния солитонов.

\section{3. ДИФФЕРЕНЦИАЛЬНАЯ НЕЛИНЕЙНАЯ ФОРМУЛА СУПЕРПОЗИЦИИ}

В данном разделе рассматривается $(1+1)$-мерная система, заданная скалярным уравнением

$$
u_{t t}+u_{t x x x}+u_{x x} u_{t}+u_{x} u_{t x}=0
$$

Нас интересует как получение ее редукций подобия при помощи метода групп Ли, так и построение нелинейной формулы суперпозиции. Уравнение (15) получается при редукции к $1+1$ измерениям частного случая уравнения Нижника-Новикова-Веселова [7], а также уравнения, рассмотренного Ито [8]. Его пара Лакса и ПД приведены в [9]. 
Следуя классическому методу групп Ли [10], рассмотрим однопараметрическую группу Ли инфинитезимальных преобразований переменных $(x, t, u)$ вида

$$
\begin{aligned}
x & \rightarrow x+\epsilon \xi(x, t, u)+O\left(\epsilon^{2}\right), \\
t & \rightarrow t+\epsilon \tau(x, t, u)+O\left(\epsilon^{2}\right), \\
u & \rightarrow u+\epsilon \phi(x, t, u)+O\left(\epsilon^{2}\right),
\end{aligned}
$$

где $\epsilon$ - параметр группы. Условие инвариантности рассматриваемого УЧП при преобразовании (16) приводит к переопределенной системе линейных уравнений для инфинитезимальных генераторов $\xi(x, t, u), \tau(x, t, u)$ и $\phi(x, t, u)$. При этом ассоциированная алгебра Ли состоит из векторных полей вида

$$
\mathbf{v}=\xi(x, t, u) \frac{\partial}{\partial x}+\tau(x, t, u) \frac{\partial}{\partial t}+\phi(x, t, u) \frac{\partial}{\partial u},
$$

и при условии, что инфинитезимальные генераторы известны, переменные симметрии для каждой редукции могут быть найдены путем решения характеристических уравнений

$$
\frac{d x}{\xi(x, t, u)}=\frac{d t}{\tau(x, t, u)}=\frac{d u}{\phi(x, t, u)} .
$$

Для уравнения (15) инфинитезимальные генераторы имеют вид

$$
\xi=c_{0} x+c_{1}, \quad \tau=3 c_{0} t+c_{2}, \quad \phi=-c_{0} u+c_{3},
$$

где $c_{0}, c_{1}, c_{2}$ и $c_{3}$ - произвольные постоянные. Отметим, что так называемые неклассические симметрии [11] (c $\tau \neq 0)$ приводят к тем же инфинитезимальным генераторам, что и классические симметрии. Инфинитезимальные генераторы (19) определяют две различные симметрийные редукции для уравнения (15), соответствуюшие нулевым и ненулевым значениям $c_{0}$. Рассмотрим их более подробно.

1. Случай $c_{0}=0$. В этом случае можно считать $c_{2}=1$ и положить $c_{1}=\alpha$ и $c_{3}=$ $\alpha(\beta-\alpha)$. Решая характеристические уравнения (18), находим редукцию типа бегушей волны

$$
u(x, t)=\alpha(\beta-\alpha) t+\frac{\beta}{2}(x-\alpha t)-\omega(z), \quad z=x-\alpha t .
$$

Дважды проинтегрировав получающееся в результате ОДУ, получаем

$$
P_{z}^{2}=\frac{2}{3}\left(P^{3}+A P+B\right),
$$

где $P(z)=\omega_{z}$, а $A$ и $B$ - постоянные интегрирования. В общем случае решениями этого уравнения являются эллиптические функции, но при некоторых значениях постоянных интегрирования имеются решения специального вида. При $A=B=0$ получаем рациональное решение

$$
P(z)=\frac{6}{(z+\delta)^{2}},
$$


из которого при помоши редукции подобия (20) получается простое решение уравнения (15). При $A=-12 b^{4}$ и $B=16 b^{6}$ имеем решение

$$
P(z)=2 b^{2}\left\{1-3 \operatorname{sech}^{2}[b(z+\delta)]\right\}
$$

из которого получается решение исходного УЧП (15)

$$
u(x, t)=C+\alpha(\beta-\alpha) t+\left(\frac{\beta}{2}-2 b^{2}\right)(x-\alpha t)+6 b \operatorname{th}[b(x-\alpha t+\delta)],
$$

где $C$ - постоянная интегрирования. Это решение соответствует односолитонному решению уравнения (15).

2. Случай $c_{0} \neq 0$. Не теряя общности, положим $c_{0}=1$ и $c_{1}=c_{2}=c_{3}=0$. Ассоциированные с этой скейлинговой редукцией переменные подобия имеют вид

$$
u(x, t)=\omega(z) t^{-1 / 3}, \quad z=x t^{-1 / 3},
$$

и для $\omega(z)$ возникает ОДУ

$$
z^{2} \omega_{z z}+6 z \omega_{z}+4 \omega-3 z \omega_{z z z z}-12 \omega_{z z z}-6 z \omega_{z} \omega_{z z}-3 \omega \omega_{z z}-6 \omega_{z}^{2}=0 .
$$

Проинтегрировав это ОДУ четвертого порядка, получаем

$$
3 z \omega_{z z z}+3 z \omega_{z}^{2}+9 \omega_{z z}+3 \omega \omega_{z}-z^{2} \omega_{z}-4 z \omega-a=0,
$$

где $a$ - постоянная интегрирования. При замене независимых и зависимых переменных

$$
\omega(z)=\frac{9}{z} m(y)+\frac{1}{3} z^{2}-\frac{1}{4 z}, \quad y=z^{3 / 2}
$$

оно переходит в ОДУ

$$
m_{y y y}+\frac{m_{y y}}{y}-\frac{4}{y^{2}} m m_{y}+\frac{6}{y} m_{y}^{2}+\frac{16}{27} \frac{y m_{y}-m}{y}-\frac{1}{2} \frac{A_{2}}{y}=0,
$$

где $A_{2}$ связано с $а$ соотношением

$$
a=\frac{3}{2}\left(5+\frac{243}{8} A_{2}\right)
$$

Это уравнение имеет первый интеграл [12]

$$
m_{y y}^{2}=-\frac{4}{y^{2}}\left[\left(y m_{y}-m\right) m_{y}^{2}+\frac{4}{27}\left(y m_{y}-m\right)^{2}-\frac{A_{2}}{4}\left(y m_{y}-m\right)-\frac{A_{4}}{4}\right],
$$


где $A_{4}$ - новая постоянная интегрирования. При преобразовании

$$
\begin{gathered}
m=\frac{1}{4 n}\left(\frac{y n_{y}}{n-1}-n\right)^{2}-\frac{1}{4}(1-\alpha)^{2}(n-1)-\frac{\beta}{2} \frac{n-1}{n}+\frac{\gamma y}{4} \frac{n+1}{n-1}+\frac{\delta}{2} \frac{y^{2} n}{(n-1)^{2}}, \\
m_{y}=-\frac{y}{4 n(n-1)}\left[n_{y}-\alpha \frac{n(n-1)}{y}\right]^{2}-\frac{\beta}{2 y} \frac{n-1}{n}-\frac{1}{4} \gamma-\frac{\delta}{2} \frac{n}{n-1} y
\end{gathered}
$$

уравнение (31) может перейти в два частных случая пятого уравнения Пенлеве (PV) (см. [13]) по переменной $n(y)$ :

$$
n_{y y}=\left(\frac{1}{2 n}+\frac{1}{n-1}\right) n_{y}^{2}-\frac{n_{y}}{y}+\frac{(n-1)^{2}}{y^{2}}\left(\frac{1}{2} \alpha^{2} n+\frac{\beta}{n}\right)+\gamma \frac{n}{y}+\delta \frac{n(n+1)}{n-1}
$$

соответствующих значениям $\delta=8 / 27, \beta=-(1-\alpha)^{2} / 2$ и $\delta=8 / 27, \gamma=0$. Константы $A_{2}$ и $A_{4}$ связаны с параметрами $\mathrm{PV}$ соотношениями

$$
A_{2}=\frac{1}{4} \gamma^{2}+\frac{32}{27} \beta, \quad A_{4}=-\frac{1}{2} \beta \gamma^{2}
$$

в первом случае и

$$
A_{2}=-\frac{8}{27}\left[(1-\alpha)^{2}-2 \beta\right], \quad A_{4}=-\frac{1}{27}\left[(1-\alpha)^{2}+2 \beta\right]^{2}
$$

во втором случае. Интересно, что, как отмечено в [13], оба эти частных случая PV можно свести к третьему уравнению Пенлеве (PIII). Это означает, что после скейлинговой редукции решение уравнения (15) можно выразить через решения пятого и третьего уравнений Пенлеве.

Оставшаяся часть данного раздела посвящена проблеме построения нелинейной формулы суперпозиции для уравнения (15). Это уравнение возникает как условие согласования для пары Лакса четвертого порядка

$$
\begin{gathered}
\psi_{x x x x}=-u_{x} \psi_{x x}+\left(\lambda-u_{x x}\right) \psi_{x}+\frac{1}{3} u_{t} \psi \\
\psi_{t}=-\psi_{x x x}-u_{x} \psi_{x}
\end{gathered}
$$

где $\lambda$ - спектральный параметр. ПД имеет вид

$$
\tilde{u}=u+6 \frac{\psi_{x}}{\psi}
$$

где $\tilde{u}$ и $u$ - два различных решения уравнения (15), связанных посредством собственной функции $\psi$ пары Лакса. Пользуясь ПД (37), можно получать решения при помощи итераций, отыскивая на каждом шаге собственную функцию пары Лакса (36). Однако этот процесс можно упростить при помоши теоремы о перестановочности, позволяюшей понизить порядок возникающих ОДУ. 
ПБ для уравнения (15) нетрудно получить, исключая собственную функцию $\psi$ из пары Лакса и ПД (37). Результатом является пара уравнений

$$
\begin{gathered}
p_{x x x}+\frac{2}{3} p p_{x x}+\frac{1}{2} p_{x}^{2}+\frac{1}{6} p^{2} p_{x}+\frac{1}{216} p^{4}+u_{x} p_{x}+\frac{1}{6} u_{x} p^{2}+u_{x x} p-\lambda p-2 u_{t}=0 \\
p_{t}+p_{x x x}+\frac{1}{2} p p_{x x}+\frac{1}{12} p^{2} p_{x}+\frac{1}{2} p_{x}^{2}+u_{x} p_{x}+u_{x x} p=0
\end{gathered}
$$

где $p=\tilde{u}-u$. Чтобы построить нелинейную формулу суперпозиции, предположим, что два решения $u_{j, 1}$ и $u_{j, 2}$ уравнения (15) построены на основе одного и того же исходного решения $\left(u_{j-1}, v_{j-1}\right)$ при помощи преобразований с различными спектральными параметрами $\lambda_{1}$ и $\lambda_{2}$, соответственно. Тогда имеем две различные копии уравнения (38): одну с $p=u_{j, 1}-u_{j-1}$ и $\lambda=\lambda_{1}$, а другую с $p=u_{j, 2}-u_{j-1}$ и $\lambda=\lambda_{2}$ (для обеих копий $u$ в уравнении $(38)$ равно $u_{j-1}$ ). Построим теперь еше одно решение $u_{j+1,12}$, исходя из $u_{j, 1}$ и $\lambda_{2}$, а также решение $u_{j+1,21}$, исходя из $u_{j, 2}$ и $\lambda_{1}$. Снова имеем две копии уравнения (38): одну с $p=u_{j+1,12}-u_{j, 1}$ и $\lambda=\lambda_{2}$, а другую с $p=u_{j+1,21}-u_{j, 2}$ и $\lambda=\lambda_{1}$. Воспользуемся теперь теоремой о перестановочности, утверждающей, что $u_{j+1,21}=u_{j+1,12}$ (как и в предыдушем примере, полагаем $\left.u_{j+1,21}=u_{j+1,12}=u_{j+1}\right)$. Тогда можно исключить все производные решений третьего порядка по пространственной переменной из четырех копий уравнения (38). Вид пространственной части ПБ (38) таков, что производные $u_{j, 1}$ и $u_{j, 2}$ по времени входят в получающиеся в результате уравнения, однако их можно исключить с помощью временно́й части ПБ (39).

Полагая $v_{j+1}=u_{j+1}-u_{j-1}, v_{j, 1}=u_{j, 1}-u_{j-1}$ и $v_{j, 2}=u_{j, 2}-u_{j-1}$, получаем нелинейное дифференциальное уравнение второго порядка для $v_{j+1}$ :

$$
\begin{aligned}
& \left(v_{j+1}\right)_{x x}+\frac{1}{2}\left(v_{j+1}\right)\left(v_{j+1}\right)_{x}+\frac{1}{36}\left(v_{j+1}\right)^{3}+\frac{1}{6} A_{2}(x, t)\left[\left(v_{j+1}\right)^{2}+6\left(v_{j+1}\right)_{x}\right]+ \\
& \quad+A_{1}(x, t)\left(v_{j+1}\right)+6 A_{0}(x, t)=0
\end{aligned}
$$

где

$$
\begin{aligned}
A_{2}(x, t)= & -\frac{1}{4}\left(v_{j, 1}+v_{j, 2}\right), \\
A_{1}(x, t)= & \frac{1}{36}\left[\left(v_{j, 1}\right)^{2}+\left(v_{j, 2}\right)^{2}+v_{j, 1} v_{j, 2}\right]- \\
& -\frac{1}{2} \frac{\left(v_{j, 2}-v_{j, 1}\right)_{x x}+3\left(\lambda_{2}-\lambda_{1}\right)}{\left(v_{j, 2}-v_{j, 1}\right)}+\frac{1}{2}\left(u_{j-1}\right)_{x}, \\
A_{0}(x, t)= & -\frac{1}{4}\left(\lambda_{2}+\lambda_{1}\right) .
\end{aligned}
$$

При замене переменных

$$
v_{j+1}=6 \frac{P_{x}}{P}
$$

уравнение (40) переходит в линейное уравнение третьего порядка

$$
P_{x x x}+A_{2}(x, t) P_{x x}+A_{1}(x, t) P_{x}+A_{0}(x, t) P=0 .
$$


Таким образом, при наличии трех решений $u_{j-1}, u_{j, 1}, u_{j, 2}$ уравнения (15) для нахождения четвертого решения $u_{j+1}$ достаточно решить линейное уравнение третьего порядка. Отметим, что для итерационной процедуры, использующей ПД и пару Лакса, требуется решение линейного ОДУ четвертого порядка.

В качестве простейшего примера описанного процесса построим двухсолитонное решение уравнения (15). Для этого получим прежде всего односолитонное решение, взяв в качестве исходного простое постоянное решение $u_{0}$ и найдя собственную функцию пары Лакса $\psi$. Односолитонное решение имеет вид

$$
u_{1 \mathrm{sol}}=u_{0}+6\left\{\ln \left(1+e^{k\left(x-k^{2} t+\delta\right)}\right\}_{x}\right.
$$

где $\lambda=k^{3}$. При $\alpha=\beta=k^{2}, b=k / 2$ и $C=3 k+u_{0}$ оно совпадает с решением (24), найденным при помоши симметрийного анализа. В уравнении (43) положим $u_{j-1}=u_{0}$, а в качестве $u_{j, 1}$ и $u_{j, 2}$ возьмем две копии односолитонного решения (44) с двумя различными значениями $k$, например $k_{1}$ и $k_{2}$, и двумя различными значениями $\delta$, например $\delta_{1}$ и $\delta_{2}$. Частное решение ОДУ (43) имеет вид

$$
P=1+e^{\eta_{1}}+e^{\eta_{2}}+a e^{\eta_{1}+\eta_{2}}
$$

где $\eta_{i}=k_{i}\left(x-k_{i}^{2} t+\gamma_{i}\right)$ при $i=1,2$, коэффициент $a$ при перекрестном члене определяется выражением

$$
a=\frac{\left(k_{1}^{2}+k_{2}^{2}+k_{1} k_{2}\right)\left(k_{1}-k_{2}\right)^{2}}{\left(k_{1}^{2}+k_{2}^{2}-k_{1} k_{2}\right)\left(k_{1}+k_{2}\right)^{2}},
$$

а константы $\gamma_{i}-$ соотношениями

$$
e^{k_{1} \gamma_{1}}=-\frac{1}{a} \frac{k_{1}-k_{2}}{k_{1}+k_{2}} e^{k_{1} \delta_{1}}, \quad e^{k_{2} \gamma_{2}}=\frac{1}{a} \frac{k_{1}-k_{2}}{k_{1}+k_{2}} e^{k_{2} \delta_{2}}
$$

Выражение (45) для $P$ и формула (42) дают двухсолитонное решение уравнения (15).

\section{4. ВЫВОДЫ}

Мы рассмотрели итерацию ПД для двух интегрируемых систем. Применение теоремы Бианки о перестановочности к соответствуюшим ПБ позволило вывести нелинейные формулы суперпозиции, значительно упрощающие итерационное построение решений. Было показано, что для получения четвертого решения этой системы через три предыдущих, вместо решения на каждом шаге линейных дифференциальных уравнений второго и четвертого порядка для собственной функции пары Лакса, можно решать соответственно линейное алгебраическое уравнение и дифференциальное уравнение третьего порядка. Более того, для одной из двух рассматриваемых систем были получены группы Ли симметрий и соответствующие редукции подобия. В частности, была получена скейлинговая редукция, позволяюшая выразить решение данной системы в терминах решений пятого и третьего уравнений Пенлеве.

Благодарности. Данное исследование частично поддержано DGICYT (контракт № $\mathrm{BFM} 2002-02609)$. 


\section{Список литературы}

[1] P. G. Drazin, R.S. Johnson. Solitons: An Introduction. Cambridge: Cambridge Univ. Press, 1989.

[2] P. R. Gordoa. J. Math. Phys. 2000. V. 41. P. 4713-4731.

[3] R. Hirota, J. Satsuma. J. Phys. Soc. Japan. 1978. V. 45. P. 1741-1750; X. B. Hu. Acta Math. Appl. Sinica. 1988. V. 4. P. 46-54; X. B. Hu, Y. Li. J. Phys. A. 1991. V. 24. P. 3205.

[4] A. Pickering. J. Math. Phys. 1996. V. 37. P. 1894-1927.

[5] L. J. F. Broer. Appl. Sci. Res. 1975. V. 31. P. 377-395; D. J. Kaup. Progr. Theor. Phys. 1975. V. 54. P. 72-78, 396-408; M. Jaulent, J. Miodek. Lett. Math. Phys. 1976. V. 1. P. 243-250; L. Martínez Alonso. J. Math. Phys. 1980. V. 21. P. 2342-2349; B. A. Kupershmidt. Comm. Math. Phys. 1985. V. 99. P. 51-73.

[6] P. R. Gordoa, J. M. Conde. Phys. Lett. A. 2002. V. 295. P. 287-298.

[7] Л. П. Нижник. ДАН СССР. 1980. Т. 254. С. 332-335; А. П. Веселов, С. П. Новиков. ДАН СССР. 1984. Т. 279. С. 20-24; 784-788.

[8] M. Ito. J. Phys. Soc. Japan. 1980. V. 49. P. 771-778.

[9] B. Konopelchenko, W. Oevel. Publ. RIMS. 1993. V. 29. P. 581-666; P. G. Estévez, S. B. Leble. Acta Appl. Math. 1995. V. 39. P. 277-294; P. A. Clarkson, E. L. Mansfield. Math. Comput. Simulation. 1997. V. 43. P. 39-55; A. P. Fordy, A. Pickering. Generalizing painlevé truncation: Expansions in Riccati pseudopotentials. In: Symmetries and Integrability of Difference Equations. Proc. of the 2nd Int. Conf. (Caterbury, UK, July 1-5, 1996). Lond. Math. Soc. Lect. Note Ser. 255. Eds. P. A. Clarkson, F. Nijhoff. Cambridge: Cambridge Univ. Press, 1999. P. 287-298.

[10] G. W. Bluman, J. D. Cole. Similarity Methods for Differential Equations. Appl. Math. Sci. V. 13. Berlin: Springer, 1974; G. W. Bluman, S. Kumei. Symmetries and Differential Equations. Appl. Math. Sci. V. 81. Berlin: Springer, 1989; П. Олвер. Приложения групп Ли к дифференциальным уравнениям. М.: Мир, 1989; H. Stephani. Differential Equations: Their Solution Using Symmetries. Cambridge: Cambridge Univ. Press, 1989.

[11] G. W. Bluman, J. D. Cole. J. Math. Mech. 1969. V. 18. P. 1025-1042.

[12] C. M. Cosgrove. Stud. Appl. Math. 2000. V. 104. P. 171-228.

[13] C. M. Cosgrove, G. Scoufis. Stud. Appl. Math. 1993. V. 88. P. 25-87. 